first 24 hours of life in 8 patients; these included all patients with a postnatal diagnosis of truncus arteriosus. Four patients required mechanical ventilation. Whilst in the neonatal intensive care unit, 3 babies developed heart failure and were treated with diuretics and 2 developed necrotising enterocolitis which was managed conservatively. Median length of stay before transfer to our local surgical cardiology centre was 3 days. Thirteen patients (87\%) underwent common arterial trunk repair. $10(77 \%)$ babies survived to date. Two patients died in the initial post-operative period and one patient at 14 months of age. The remaining two patients did not receive any surgical intervention and subsequently died (4 and 11 days old). Of the 10 babies who survived, the median length of hospital stay post operatively was 20 days.

Conclusion Truncus arterisus is a rare conotruncal defect. The majority of these were detected antenatally. They are likely to require respiratory support but the surgical outcomes are good. The following information can be used when counselling parents antenatally and managing their expectations.

\section{P449 PROBIOTICS AND RETINOPATHY OF PREMATURE: SYSTEMIC REVIEW}

${ }^{1}$ Reza Saeidi*, ${ }^{2}$ Azra Izanloo. ${ }^{1}$ Department of Pediatrics, Mashhad University of Medical Sciences, Mashhad, Iran, Islamic Republic of; ${ }^{2}$ Razavi Cancer Research Center, Razavi Hospital, Imam Reza International University, Mashhad, Iran, Islamic Republic of

\subsection{6/archdischild-2019-epa.785}

Introduction As we know, ROP is a multifactorial disease, and several preventable factors are dependent on increased ROP. Thus, we decided to undertake a systematic review to study the effects of probiotics on the prevalence and severity of ROP.

Method We searched studies in the PubMed, Cochrane collaborative library and Google Scholar at all levels until January 30, 2019. The papers were evaluated independently by two reviewers according to the predefined criteria and relevant data were extracted. The initial complications of ROP and its severity and stage were analyzed.

Results Of the 780 extracted studies, only 13 studies were relevant, none of which had directly investigated the effect of probiotics on the prevalence of ROP. Athorough study of the articles revealed that 9 studies had examined the effect of probiotics on the severity of ROP without reporting any significant correlation. Moreover, 4 studies had assessed the effect of probiotics on the stage of ROP, with none of them reporting a significant difference. A meta-analysis published in 2017 suggested that the use of probiotics significantly reduces the risk of infection without affecting the severity and stage of ROP.

Conclusion According to the results of the study, a challenging problem in clinical trials is that poor infant growth can be a predictor of ROP and proper nutrition can improve infant growth. Hence, further studies are required to determine whether probiotics have an indirect or direct effect on the incidence or severity of ROP or not.
P450 POOR OUTCOMES PREDICTORS IN NEWBORNS FROM MOTHERS WITH GESTATIONAL DIABETES

Anastasiya Vanyarkina*, Alla Petrova, Lyubov Rychkova. Scientific Centre for Family Health and Human Reproduction Problems, Irkutsk, Russian Federation

\subsection{6/archdischild-2019-epa.786}

Background Rising of gestational diabetes (GD) incidence along with obesity, leads to increasing both pregnancy and neonatal complications. In utero exposure to hyperglycaemia increases the risk of future obesity and type 2 diabetes development.

The aim was to evaluate influence of metabolic disorders and body mass index (BMI) in women with GD on the risk of diabetic fetopathy development in their newborns.

Materials and methods Monocentric, ambispective, observational study was conducted at Tertiary Perinatal Centre, Irkutsk, Russia, between January 2018 and January 2019. It included 34 newborns from mothers with GD. Inclusion criterion was confirmed GD in the mother. Pregnancy complications, perinatal outcomes in diabetic mothers and their newborns followup data were collected and analyzed.

Results At birth, mean weight was 2647 grams (IQR 9204420). Mean gestational age was 38 weeks (IQR 27-41). Among newborns, 52.9\% was premature and 5.8\% was small for gestational age. Symptomatic diabetic fetopathy (DF) was revealed in 17 infants (DF group), and 17 infants did not have DF (controls).

Mothers of newborns with fetopathy were tested on GD at 31 (IQR 30-32) week of pregnancy, and mothers of controls $(\mathrm{p}=1.0)$ - at 25 (IQR 20-30) week.

It was found that mothers of newborns with DF 6 times oftener had an elevated BMI at first pregnancy consultation than mothers of controls - 12 vs 2 , respectively $(\mathrm{OR}=3.4$; 95\% CI: 1.2-5.5), and they had excessive weight gain during pregnancy 3.5 times oftener than those without DF - 14 vs 4 $(\mathrm{OR}=4.1 ; 95 \% \mathrm{CI}: 1.0-6.7)$, respectively.

GD was associated with hypertensive disorders of pregnancy (including pre-eclampsia, pregnancy-induced hypertension) in most mothers of DF group, comparing to controls (82.3\% versus $47 \%$; $\mathrm{p}=0.03)$.

Ultrasonography detected excessive growth of the fetus $(11.7 \% ; p=0.2)$ and polyhydramnios $(29.4 \% ; p=0.3)$ in mothers of DF group.

Mothers of DF group had caesarean sections 5 times oftener than mothers of controls: 10 vs $2 \quad(p=0.004)$, respectively.

Macrosomia, neonatal hypoglycaemia and hypocalcaemia were common symptoms among DF newborns compared with controls: $47 \%$ vs $11.7 \% \quad(\mathrm{p}=0.02), 100 \%$ vs $0 \quad(\mathrm{p}<0.001)$, $58.8 \%$ vs $5.8 \%(\mathrm{p}=0.001)$, respectively.

Conclusions We showed that metabolic disorders, high baseline BMI during pregnancy, and late GD detection in diabetic mothers are leading causes of poor perinatal outcomes and symptomatic DF in infants. Implementation of GD screening program, informing about baseline metabolic disorders in women and baseline BMI during pregnancy can prevent both maternal and infant health outcomes. 